\title{
Emergence of Celiac Disease and Gluten-related Disorders in Asia
}

\author{
Srikant Mohta, Mahendra S Rajput, Vineet Ahuja, and Govind K Makharia* \\ Department of Gastroenterology and Human Nutrition, All India Institute of Medical Sciences, New Delhi, India
}

Celiac disease (CeD) is a systemic, immune-mediated enteropathy, which is triggered by gluten protein in genetically susceptible individuals. $\mathrm{CeD}$, once thought to be an uncommon disease, is now recognized to affect approximately 40-60 million people globally. While CeD is now well reported from a few Asian countries such as India, China, Pakistan, and Middle Eastern countries; it is still believed to be uncommon in the rest of Asia. Gluten-related diseases other than CeD, like non-celiac gluten sensitivity (NCGS) are also emerging globally. CeD and NCGS may present with either intestinal or extra-intestinal symptoms, and a proportion of them have overlapping symptoms with irritable bowel syndrome. Hence, many of them are misdiagnosed as having irritable bowel syndrome in clinical practice. In this review, we discuss the emergence of CeD and other gluten-related disorders, both globally and in Asia, the overlapping manifestations between gluten-related disorders and irritable bowel syndrome, and the challenges associated with diagnosis and management of CeD in Asia.

(J Neurogastroenterol Motil 2021;27:337-346)

\section{Key Words}

Epidemiology; Glutens; India; Irritable bowel syndrome; Non-celiac gluten sensitivity

\section{Introduction}

Celiac disease $(\mathrm{CeD})$ is a systemic, small intestinal, immunemediated enteropathy which is triggered and maintained by gluten protein present in cereals such as wheat, barley and rye. ${ }^{1}$ Until a few decades ago, $\mathrm{CeD}$ was considered to be an uncommon disease and believed to be limited to a certain part of Europe. ${ }^{2}$ In the absence of a good diagnostic or screening blood test, it was hard to make a diagnosis of $\mathrm{CeD}$ which required 3 sequential intestinal mucosal biopsies. With the advent of celiac-specific serological test for the screening and the diagnosis, the epidemiology of $\mathrm{CeD}$ has changed globally over the past 2 decades. ${ }^{3}$ Furthermore, simplification of the diagnostic criteria and widespread use of celiac-specific serological tests have not only led to increase in the recognition of $\mathrm{CeD}$ worldwide, but also enabled us to assess the accurate prevalence of $\mathrm{CeD}$ in the general population. ${ }^{2,4,5}$ Initial studies of the prevalence in the general population originated from the European countries ${ }^{6,7}$ and subsequently regions with a predominant Caucasian population such as North America, Australia, and South American countries have also reported occurrences of $\mathrm{CeD} .^{8-10}$ In recent times, $\mathrm{CeD}$ is recognized in many non-Caucasian populations including Asian and African countries. $^{11,12}$

With $60 \%$ of the world's population, Asia is presently at the helm of understanding the epidemiology of $\mathrm{CeD}$. In spite of an

Received: June 22, 2020 Revised: August 13, 2020 Accepted: April 22, 2021

() This is an Open Access article distributed under the terms of the Creative Commons Attribution Non-Commercial License (http://creativecommons. org/licenses/by-nc/4.0) which permits unrestricted non-commercial use, distribution, and reproduction in any medium, provided the original work is properly cited.

*Correspondence: Govind K Makharia, MD, DM, DNB, MNAMS

Department of Gastroenterology and Human Nutrition, All India Institute of Medical Sciences, Ansari Nagar, New Delhi 110029 , India

Tel: +91-9910678476, Fax: +91-11-26588091, E-mail: govindmakharia@gmail.com 
Table 1. Differentiating Features Between Celiac Disease, Non-celiac Gluten Sensitivity and Wheat Allergy

\begin{tabular}{|c|c|c|c|}
\hline & Celiac disease & NCGS & Wheat allergy \\
\hline Prevalence & $0.5-2.0 \%$ & $0.6-6.0 \%$ & $0.4-1.0 \%$ in children \\
\hline Age of presentation & Childhood and adulthood & Mostly adulthood (3rd-4th decade) & Mostly childhood (1st decade) \\
\hline Sex & $\mathrm{F}>\mathrm{M}(1.3: 1-2: 1)$ & $\mathrm{F}>\mathrm{M}(3: 1-5.4: 1)$ & $\mathrm{M}>\mathrm{F}$ \\
\hline $\begin{array}{l}\text { Onset of symptoms after } \\
\text { gluten exposure }\end{array}$ & Weeks-years & Hours-days & Minutes-hours \\
\hline Pathogenesis & Autoimmunity (adaptive immunity) & $\begin{array}{l}\text { Poorly understood, role of both in- } \\
\text { nate and adaptive immunity }\end{array}$ & $\begin{array}{l}\text { Allergic immune response ( } \mathrm{IgE} \\
\text { antibody against wheat proteins) }\end{array}$ \\
\hline \multicolumn{4}{|l|}{ Clinical features } \\
\hline Intestinal & $\begin{array}{l}\text { Chronic diarrhea, abdominal pain, } \\
\text { bloating }\end{array}$ & Diarrhea, abdominal pain, bloating & Less often \\
\hline \multirow[t]{7}{*}{ Extra-intestinal } & Anemia & Tiredness & More often \\
\hline & Osteoporosis & Lack of well-being, headache, & Skin rashes urticaria Angioedema, \\
\hline & Neurological symptoms (ataxia, & foggy mind, fatigue & asthma, cough \\
\hline & peripheral neuropathy) & Numbness of limbs & \\
\hline & Growth retardation & Joint/muscle pain & \\
\hline & & Fainting & \\
\hline & & Oral/tongue lesions & \\
\hline \multirow[t]{3}{*}{ Serology } & IgA anti-tTG $\mathrm{Ab}$ & IgG Anti-gliadin Ab & $\mathrm{IgE}$ antibody to wheat protein \\
\hline & IgA anti-EMA & & \\
\hline & IgA anti-DGP $\mathrm{Ab}$ & & \\
\hline Gastroduodenoscopy & $\begin{array}{l}\text { Scalloping, grooving of duodenal } \\
\text { folds }\end{array}$ & Normal & Normal \\
\hline Histology & $\begin{array}{l}\text { Various grades of villous atrophy } \\
\text { with crypt hyperplasia }\end{array}$ & $\begin{array}{l}\text { Normal/increased intra-epithelial } \\
\text { lymphocytes }\end{array}$ & Normal \\
\hline Treatment & Gluten-free diet & Gluten free diet & Wheat restriction \\
\hline Spontaneous resolution & No & Not known & $65 \%$ by 12 years of age \\
\hline
\end{tabular}

NCGS, non-celiac gluten sensitivity; F, female; M, male; Ab, antibody; tTG, tissue transglutaminase; EMA, endomysial antibody; DGP, deamidated gliadin peptide; FODMAP, fermentable oligosaccharides, disaccharides, monosaccharides, and polyols.

increase in the publications on $\mathrm{CeD}$ from Asia, there is a dearth of data on population-based prevalence from most nations, except for India and China. ${ }^{10,13-19}$

It is not only $\mathrm{CeD}$ which has caught the eye of clinicians and clinical scientists, the spectrum of wheat and or gluten-related disorders has also expanded in recent times. The widespread consumption of wheat makes the disorders related to gluten relevant for most of the world including Asia. The broad spectrum of gluten-related disorders includes:

- Autoimmune diseases: $\mathrm{CeD}$, dermatitis herpertiformis, and gluten ataxia

- Non-autoimmune, non-allergic disorder: non-celiac gluten sensitivity (NCGS)

- IgE mediated allergic reactions: classic wheat allergy, wheatdependent exercise induced anaphylaxis, and occupational asthma (baker's asthma)

These gluten-related disorders differ in their pathophysiology and their clinical symptomatology, which are summarized in Table $1 .{ }^{20-26}$

The phenotypic spectrum of $\mathrm{CeD}$ is diverse including classical and non-classical $\mathrm{CeD}$. Those with typical signs and/or symptoms of malabsorption such as diarrhea, weight loss, vitamin deficiencies, or malnutrition are classified as classical CeD. Patients with non-classic $\mathrm{CeD}$ are difficult to recognize as they present with non-gastrointestinal symptoms such as short stature, anemia, amenorrhea, or osteopenia/osteoporosis. A significant fraction of patients is asymptomatic or have non-specific mild complaints which are managed symptomatically for prolonged duration. These patients with $\mathrm{CeD}$ having mild gastrointestinal symptoms may fulfill the criteria for the diagnosis of functional gastrointestinal diseases, mostly irritable bowel syndrome (IBS). This may lead to a missed diagnosis and may be treated as IBS. 


\section{Methods}

We searched the PubMed using the terms "celiac disease," "gluten sensitivity," or "non-celiac gluten sensitivity," "irritable bowel syndrome" or "functional gastrointestinal disease," "epidemiology," "prevalence," "risk factors," "clinical manifestations," or "overlap." Abstracts were screened and articles which had relevant information on celiac disease or its associated disorders in any of the Asian countries were included. The search was extended by using the references of selected recent articles and systematic reviews or meta-analysis.

\section{Changing Epidemiology of Celiac Disease -}

\section{Global Prevalence of Celiac Disease}

The prevalence of $\mathrm{CeD}$ in a population can be assessed using 2 methods. One way is to assess the seroprevalence of $\mathrm{CeD}$, which is done using celiac-specific serological tests, and the proportion of people having a positive test is defined as seroprevalence in a population or a community. The prevalence of confirmed $\mathrm{CeD}$ is assessed using a combination of serological tests specific to $\mathrm{CeD}$ and demonstration of villous abnormalities using duodenal mucosal biopsies. We, in a recent systematic review and meta-analysis of population-based studies, including 275818 subjects, have shown a pooled global seroprevalence of $\mathrm{CeD}$ to be $1.4 \%$ (95\% CI, 1.1-1.7\%). ${ }^{14}$ The global pooled prevalence of biopsy-confirmed CeD was found to be $0.7 \%$ (95\% CI, 0.5-0.9\%), meaning thereby 1 in 140 individuals have $\mathrm{CeD}$. The differences in the seroprevalence and prevalence of biopsy-confirmed $\mathrm{CeD}$ is mainly because lack of confirmation of presence of villous abnormalities in seropositive individuals by endoscopic mucosal biopsies in many studies. ${ }^{27,28}$

The prevalence of $\mathrm{CeD}$ varies from continent to continent, the highest being in Europe. On stratification of countries into quartiles based on the prevalence of biopsy-confirmed $\mathrm{CeD}$, countries with highest prevalence (76th to 100th quartile) include Argentina, Egypt, Hungary, Finland, India, New Zealand, and Sweden, and countries with lowest prevalence (0 to 25th quartile) include Brazil, Germany, Republic of San Marino, Russia, and Tunisia. ${ }^{29}$

Based on above mentioned prevalence data, approximately 40 60 million individuals have $\mathrm{CeD}$ globally and of those, the majority (83-95\%) are in developed countries, and even larger numbers in the developing countries still remain undiagnosed. ${ }^{30}$

\section{Celiac Disease in Asia}

The epidemiology of $\mathrm{CeD}$ is different in different parts of Asia due to the heterogeneity of population, genetics, economic condition, and dietary habits (Figure). ${ }^{31-36} \mathrm{~A}$ recent systematic review and meta-analysis showed that the pooled prevalence based on serological tests (IgA anti-tissue transglutaminase (tTG) antibody [Ab] and/or anti-endomysial antibodies [EMA]) of $\mathrm{CeD}$ in Asian countries was $1.6 \%$ among 47873 participants. The pooled prevalence of biopsy proven $\mathrm{CeD}$ was $0.5 \%$ in 43955 individuals. $^{37}$

In a multicenter pan-India study including 23331 healthy adults the age adjusted seroprevalence of $\mathrm{CeD}$ was $1.23 \%$ in Northern India, $0.87 \%$ in North-eastern India, and $0.10 \%$ in Southern India. This study demonstrated regional differences in the prevalence of $\mathrm{CeD}$ which was likely because of difference in the wheat (gluten) eating pattern, being highest in the Northern part of India and lowest in the South. Genetic differences like population prevalence of predisposing gene for $\mathrm{CeD}$ such as human leukocyte antigen (HLA)-DQ2 and/or HLA-DQ8 are unlikely to explain such a significant difference. ${ }^{11}$

The interest in $\mathrm{CeD}$ in China was sparked by a systematic review and meta-analysis of the predisposing genes for $\mathrm{CeD}$ done by Yuan et $\mathrm{al}^{33}$ who had predicted that $\mathrm{CeD}$ should not be uncommon in China. Two studies on the prevalence of $\mathrm{CeD}$ have been published from China after the publication of the above mentioned systematic review. In a recent cross-sectional study, 19778 Chinese youth (age 16-25 years) from 27 regions were recruited at 2 universities in Jiangxi, China, from September 2010 through October 2013. They were all tested for IgG against deamidated gliadin peptides (IgG anti-deamidated gliadin peptide), and IgA anti-tTG Ab. The prevalence was dramatically higher (12-fold) in the Northern provinces, where wheat was the staple diet. The seroprevalence of $\mathrm{CeD}$ in the Shandong province was $0.76 \%$ (95\% CI, 0.21-1.95\%), similar to the rest of the world. ${ }^{29}$ In another recent study including 2277 in-patients with gastrointestinal symptoms in 4 major ethnic groups of Xinjiang Uyghur Autonomous Region, China (1391 Han, 608 Uyghur, 146 Kazakh, and 132 Hui), the seroprevalence and prevalence of biopsy-confirmed $\mathrm{CeD}$ was found to be $1.27 \%$ (95\% CI, $0.81-1.73 \%)$ and $0.35 \%(95 \% \mathrm{Cl}, 0.11-0.59 \%)$, respectively. ${ }^{38}$ The frequency of the HLA-DQ2 and/or DQ8 haplotype ranged from $40.0-52.1 \%$ in that region. The rural parts of China, where wheat consumption was higher than the urban part, had 3 times higher prevalence of $\mathrm{CeD}(3.16 \%$ vs $0.97 \%, P<0.01)$. Interestingly, of 246 patients with diarrhea-predominant IBS in China, $2.85 \%$ were diagnosed to have $\mathrm{CeD}{ }^{39}$ These preliminary 


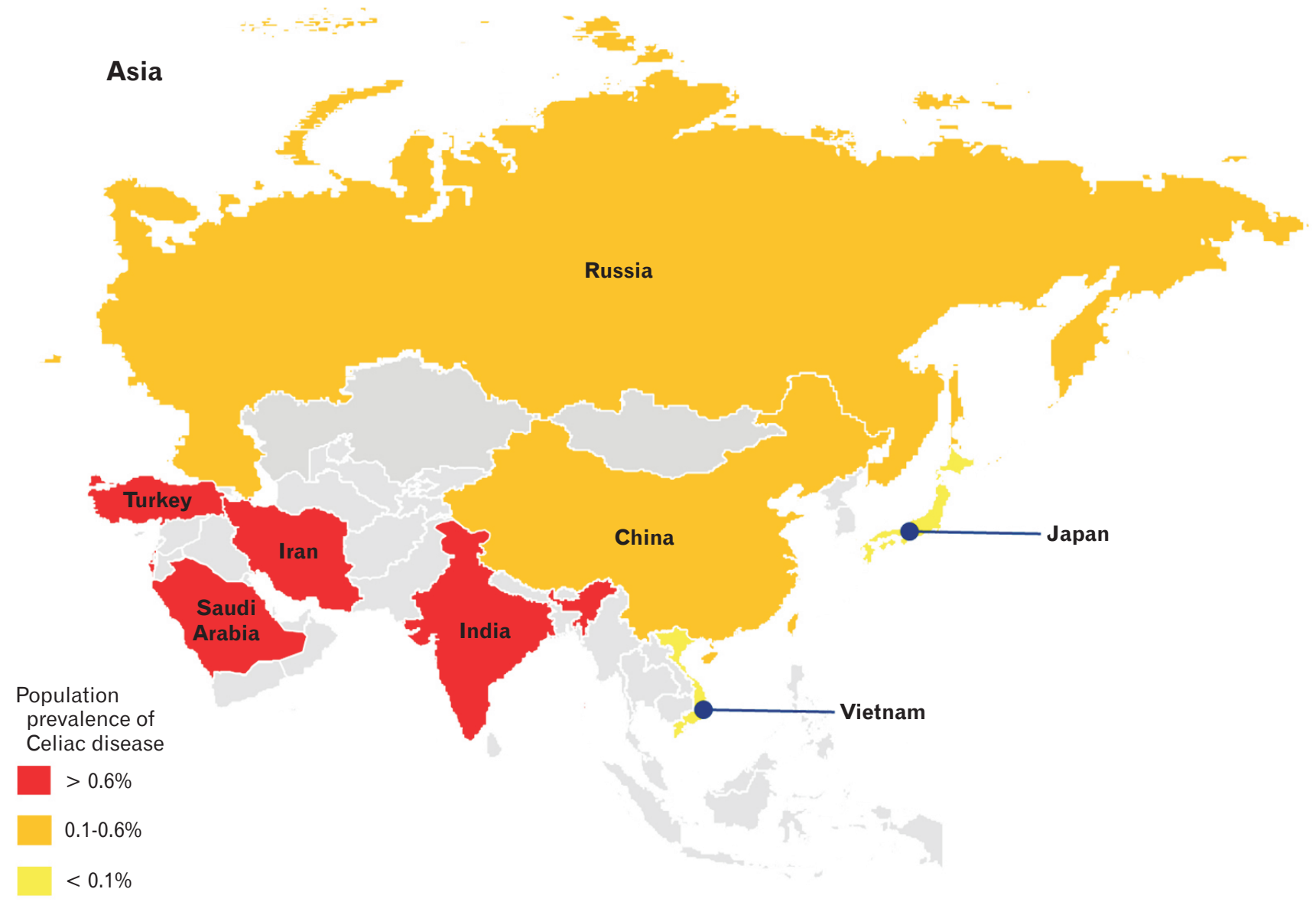

Unknown

Figure. Map of Asia showing population prevalence of Celiac disease across Asia.

studies establish the foundation for exploration of the exact prevalence of $\mathrm{CeD}$ and the regional geographical differences in China.

In a relatively small study including 562 young healthy volunteers from Malaysia, a seroprevalence of $\mathrm{CeD}$ was found to be $1.25 \%$ (95\% CI, 0.78-1.72\%). In this multi-ethnic country, all 3 ethnic group such as Malay (0.8\%), Chinese (1.7\%), and Indian (1.3\%) were affected. ${ }^{40}$

In a study from Japan, $12.8 \%$ and $13.4 \%$ of 172 patients with inflammatory bowel disease were found to have anti-tTG Ab and anti-deamidated gliadin peptide $\mathrm{Ab}$ respectively, in comparison to $1.6 \%$ and $0.5 \%$ in the same number of controls. ${ }^{34}$ Although this did not correlate well with biopsies as many patients had an increase in intraepithelial lymphocytes, none had villous atrophy. A subset of the inflammatory bowel disease patients who were put on glutenrestricted diet showed a reduction in the antibody titres, as well as improved activity scores of inflammatory bowel disease. In another study including 2008 subjects, anti-tTG Ab was found to be in a high proportion (8.0\%), none of them however were EMA positive and only 1 demonstrated changes suggestive of $\mathrm{CeD}$ on duodenal biopsy. ${ }^{41}$ Another study including 1961 Vietnamese children showed that $1.0 \%$ of them had anti-tTG ab, but none of them were EMA positive. ${ }^{42}$

Few counties in Asia have not formally reported $\mathrm{CeD}$, including Indonesia, Korea, and Taiwan. ${ }^{43,44}$

\section{Increase in the Incidence of Celiac Disease}

Not only the prevalence, but incidence of $\mathrm{CeD}$ has also increased throughout the Western world. The pooled average annual incidence of $\mathrm{CeD}$ has been rising by $7.5 \%$ (95\% CI, 5.8-9.3) per year over the past several decades. A recent systematic review reported the pooled incidence of $\mathrm{CeD}$ in women and men to be 17.4 (95\% CI, 13.7-21.1) and 7.8 (95\% CI, 6.3-9.2) per 100000 
person-years, respectively. ${ }^{45}$

The increase in the incidence in $\mathrm{CeD}$ is due to improved diagnostics and higher awareness about the disease amongst physicians and changes in our environment. Improved hygiene and a decrease in the exposure to microbes early in life has been postulated to induce an overactive immune response later in life, and thus many autoimmune disorders, including $\mathrm{CeD}{ }^{46}$

\section{Risk Factors That Might Determine Prevalence of Celiac Disease in Any Population}

The pathogenesis of $\mathrm{CeD}$ involves a complex interplay of environmental and genetic factors. Based on the prevalence of the high risk categories in these factors, hotspots of the world for $\mathrm{CeD}$ can be identified. ${ }^{47}$

The 2 most populous countries of the world, India and China, also grow the maximum amount of wheat grains. Indian's consume an average of $48.0 \mathrm{~kg} /$ person/year. ${ }^{48}$ In contradiction to usual belief, the per capita consumption of wheat flour in rural Chinese households is $59.6 \mathrm{~kg}$ and much higher than that in urban Chinese households (12.5 kg). ${ }^{33}$ Furthermore, increasing Western influence in the diet and use of commercial gluten-based foods like breads, noodles, and pasta is leading to increase consumption of gluten intake in many Asian countries. The ingestion of gluten is further accentuated by urbanization of small towns, migration to busy metropolitan cities, and culture of fast food restaurant dining. An increase in gluten ingestion is likely to increase chances of development of $\mathrm{CeD}$ in genetically susceptible populations. ${ }^{33}$

Over the years, it has become clear that $\mathrm{CeD}$ is not a monogenic disorder. The HLA alleles which have been shown to pose the highest risk for $\mathrm{CeD}$ and are found in more than $95 \%$ of $\mathrm{CeD}$ are HLA-DQ2 (HLA-DQA1*0501 and HLA-DQB1*0201) and HLA-DQ8 (HLA-DQA1*0301 and HLA-DQB1*0302). ${ }^{49-54}$ The biological plausibility of this predisposition is explained by the high affinity of deamidated gliadin peptides to HLA-DQ2 and HLA-DQ8 molecules. ${ }^{53}$ The contribution of these 2 genes is alone enough to account for $40 \%$ of the heritable risk of developing

Table 2. Spectrum of Clinical Manifestations and Associated Autoimmune Diseases in Celiac Disease

\begin{tabular}{|c|c|c|}
\hline & System & Manifestation \\
\hline \multirow[t]{3}{*}{ Classical manifestations } & Intestinal & Chronic diarrhea \\
\hline & & Bloating \\
\hline & & Generalized fatigue \\
\hline \multirow[t]{24}{*}{ Non-classical manifestations } & Cutaneous & Dermatitis herpetiformis \\
\hline & & Vitiligo \\
\hline & Endocrinological & Short stature \\
\hline & & Infertility \\
\hline & & Delayed menarche \\
\hline & & Amenorrheal \\
\hline & Hematological & Anemia \\
\hline & & Thrombocytosis \\
\hline & Hepatic & Hypertransaminesemia \\
\hline & & Autoimmune hepatitis \\
\hline & & Cirrhosis of liver \\
\hline & Muscular & Tetany \\
\hline & & Weakness \\
\hline & Neurologic & Cerebellar ataxia \\
\hline & & Peripheral neuropathy \\
\hline & & Seizures \\
\hline & Skeletal & Osteopenia, osteomalacia, and osteoporosis \\
\hline & & Pathologic fractures \\
\hline & Dental & Dental enamel defects \\
\hline & Psychiatric & Autism spectrum disorder \\
\hline & & Anxiety disorder \\
\hline & & Attention deficit hyperactive disorder \\
\hline & & Mood disorder \\
\hline & & Schizophrenia \\
\hline
\end{tabular}


CeD. ${ }^{54,55}$ Homozygosity of HLA-DQ2 depicts a gene-dose effect and is a predictor of complicated $\mathrm{CeD}$ like refractory disease or malignant transformation. ${ }^{54,55}$ It must be noted though, that approximately $40 \%$ of the Western population also carry these haplotypes without ever developing CeD, and thus HLA-DQ2 and DQ8 are necessary but not sufficient for $\mathrm{CeD}$ to develop. ${ }^{1}$

In a meta-analysis of HLA-DQ2 and -DQ8 haplotype in China, it was reported that HLA-DQ2 was more prevalent in the Northwestern than in the Southeastern populations of India which correlates with the reported prevalence of $\mathrm{CeD}$ in these regions. ${ }^{33} \mathrm{In}$ other Asian countries such as Japan, Taiwan, Korea, and Indonesia the frequency of predisposing HLA allele is low $(<5 \%){ }^{56}$

\section{Manifestations of Celiac Disease}

$\mathrm{CeD}$ has a myriad of clinical manifestations and the spectrum includes asymptomatic potential $\mathrm{CeD}$, classical $\mathrm{CeD}$, and nonclassical $\mathrm{CeD}$. The clinical features of classical and non-classical $\mathrm{CeD}$ are presented in Table $2 .^{57}$

\section{Non-celiac Gluten Sensitivity}

NCGS is an evolving entity defined by intestinal and/or extraintestinal symptoms related to wheat ingestion in individuals in whom CeD and wheat allergy has been excluded. ${ }^{57,58}$ NCGS is also termed as "gluten sensitivity," "gluten hypersensitivity," or "non-celiac wheat sensitivity." There is a wide spectrum of manifestations of NCGS, both extraintestinal and intestinal (Table 1). Epidemiological data on NCGS is still sketchy but experts believe that it is more common than $\mathrm{CeD}{ }^{59}$ The prevalence of NCGS ranges from $0.6-10.6 \%$ and this wide range of prevalence is mainly because of varying definitions of NCGS used in studies. ${ }^{59}$ Lack of data on the true prevalence of NCGS is primarily due to 2 reasons. Firstly, there is no specific biomarker for NCGS, and making of a diagnosis of NCGS requires a rigorous protocol including an initial gluten-free diet followed by double blind placebo-controlled foodchallenge with cross over. ${ }^{58}$ Secondly, many people avoid gluten for symptom relief without medical advice or consultation and rely on self diagnosis. At present, there is a dearth of data on the prevalence of NCGS in Asian populations.

\section{Overlap Between Gluten-related Disorders and Irritable Bowel Syndrome}

The clinical demarcation between IBS, CeD and NCGS is not very clear. A recent meta-analysis including 22 studies with 6991 patients with IBS has shown that 3.3\% (95\% CI, 2.3-4.5\%) of them had $\mathrm{CeD} .{ }^{60}$ Interestingly the pooled prevalence of $\mathrm{CeD}$ also varied significantly with IBS subtype. As expected, the prevalence of $\mathrm{CeD}$ was the highest in patients with IBS-D (pooled prevalence of 5.4\%; 95\% CI, 3.3-7.8\%) and mixed IBS 3.1\% (95\% CI, 1.7-5.1\%), and lowest in those with constipation-predominant IBS 1.8\% (95\% CI, 0.9-3.0\%). A couple of points merit mention here. Firstly, most of the studies, except for one, originated from secondary or tertiarycare referral centers, hence the data may not be applicable to IBS population as a whole. Secondly, 20 of 22 studies originated from Europe and Asia with pooled prevalence of $3.9 \%$ (95\% CI, 2.1$6.3 \%$ ) and $3.7 \%$ (95\% CI, 2.2-5.6\%), respectively. Studies from North America have not found an increased seroprevalence of $\mathrm{CeD}$ among patients with IBS. Thus, the prevalence of $\mathrm{CeD}$ in patients with IBS may vary with geographic regions.

Since the prevalence of $\mathrm{CeD}$ in patents with IBS is higher than the general population, especially diarrhea-predominant IBS (IBSD) and mixed IBS, these patients should be screened for CeD. ${ }^{61}$ Not only CeD, some of the patients with IBS may have NCGS, however there is no screening test for NCGS at the present point of time. While the diagnosis of $\mathrm{CeD}$ is based on a combination of a positive celiac specific serological test and demonstration of villous atrophy on intestinal mucosal biopsies, diagnosis of NCGS however requires symptom response on gluten withdrawal and reappearance of symptoms on rechallange with gluten in a double blind fashion. Furthermore, it is well known that certain foods including wheat are the triggers of symptoms in patients with IBS. ${ }^{62}$ Symptomatic improvement has been seen in a significant proportion of patients with IBS-D with the exclusion from gluten from their diet. This is supported by the reduced stool frequency with gluten-free diet in IBS-D patients $(n=45)$ in a randomized trial. ${ }^{63}$

Wheat is composed of both gluten and fermentable oligosaccharides, disaccharides, monosaccharides, and polyols (FODMAP). ${ }^{64}$ Both randomized- controlled trials and meta-analysis of randomized controlled trials have demonstrated symptomatic improvement in a third of IBS patients with low FODMAP diet. ${ }^{65}$ Since ingestion of wheat is also restricted in patients prescribed a low FODMAP diet, a low FODMAP diet leads to reduction in gluten intake because they tend to co-exist in wheat. Similarly, a diet devoid of gluten also leads to low FODMAP ingestion. ${ }^{66}$

It is still debatable as to which component of wheat causes symptoms in patients having IBS-like symptoms and NCGS. The leading candidates which are suspected are gluten and fructans. This has been explored by 2 randomized controlled trials. ${ }^{64,67}$ In 
the first double-blind cross-over trial including 37 patients with NCGS and IBS, patients were randomly assigned to high-gluten (16 g gluten/day), low-gluten ( $2 \mathrm{~g}$ gluten/day plus $14 \mathrm{~g}$ of whey), or control (16 g whey protein/day) for 1 week, following 2 weeks of low FODMAP diet. After a washout period of at least 2 weeks they were crossed over to other diet. ${ }^{67}$ In all the study subjects, gastrointestinal symptoms improved consistently and significantly during low FODMAP diet, but significantly worsened to a similar degree when they were put again on gluten or whey protein, suggesting a contribution of anticipatory "nocebo" response to gluten challenge in these individuals with perceived gluten sensitivity. In another randomized, double-blind cross-over challenge trial, 59 patients with NCGS were provided gluten, fructans, or placebo, concealed in museli bars. The patients randomized to fructans showed significantly higher overall gastrointestinal severity score than those randomized to gluten or placebo. ${ }^{64}$ Since wheat contains both gluten and FODMAP, patients reporting symptoms on wheat restriction may have obtained benefit in their symptoms because of lower intake of FODMAP (fructans) rather than gluten restriction. Another possibly implicated component of wheat in causation of is amylase trypsin inhibitors, which have a potential of activating the innate immune system via toll-like receptor 4 pathways. However, its clear role in IBS has yet to be determined. ${ }^{68}$ In addition to components of wheat, intestinal dysmotility has also been implicated in the pathogenesis of both IBS and NCGS. ${ }^{69}$

While a small proportion of patients with IBS may have $\mathrm{CeD}$, as discussed above, almost one third of patients with $\mathrm{CeD}$ who are on gluten-free diet do complain of some GI symptoms, which mimics IBS-type symptoms. ${ }^{61}$

\section{Discussion}

While $\mathrm{CeD}$ is emerging in many Asian countries, there is very limited preparedness to handle these patients in Asia. A welcome step was made when a working group of 13 members from the Asia-Pacific region and World Gastroenterology Organization reviewed relevant literature on issues specific to the Asia-Pacific region for the diagnosis and management of $\mathrm{CeD}$ and recommended possible solutions. ${ }^{70}$ Furthermore, the Asia-Pacific Association for Gastroenterology has created a formal working group on celiac disease to conduct relevant research to unravel the burden of $\mathrm{CeD}$ in Asia. We have recently reviewed the challenges in the awareness about $\mathrm{CeD}$ in Asia, the availability of the diagnostic tests, and management-related challenges such as availability of trained dieticians and availability of reliable gluten-free products in Asia. ${ }^{70}$

One of the most important priorities about $\mathrm{CeD}$ is Asia is to explore and estimate the prevalence of $\mathrm{CeD}$ in many Asian countries and increase in the awareness about this disease amongst gastroenterologists, internists, pediatricians, pathologists, and primary care physicians.

Celiac-specific serological tests are the center stage of both the screening of the suspected patient and in the diagnosis of $\mathrm{CeD}$. Currently, most of the celiac-specific serological test kits are imported from Europe and North America. These tests have their diagnostic accuracy evaluated for Caucasian populations and thus, the cut-offs of the antibody levels are determined for these populations. With a difference in the genetic make-up and the amount of gluten ingestion, the cut-offs for a positive test determined for the Caucasian population may not apply to Asian populations. In another study, we have observed different cutoff values of the IgA anti-tTG Ab test between North American and Indian patients with $\mathrm{CeD} .^{71}$

The successful management of $\mathrm{CeD}$ is primarily dependent on a combination of factors involving the understanding of disease by the patient as well as following the prescribed dietary restrictions. There is a dearth of trained dietitians in Asia to adequately counsel these patients. Due to this dearth, it is important for physicians to know more about the practical aspects of prescribing a gluten-free diet. This includes not just restriction of foods but also advising a well-balanced diet tailored to each individual patient. ${ }^{72,73}$ There is also a need for availability of reliable gluten-free food in the food supply chain and legislation for maintenance of quality control of gluten-free food in the Asian food industry.

\section{Conclusion}

In conclusion, the total number of patients with $\mathrm{CeD}$ in Asia, because of large its population, is likely to overtake the total numbers of patients in rest of the world. There is a need to recognize presence of $\mathrm{CeD}$ in Asian countries, and such Asian countries should start preparing to handle the emerging epidemic of $\mathrm{CeD}$ in Asia.

Acknowledgements: We sincerely acknowledge Indian Council of Medical Research, Department of Health Research, Government of India; and Department of Science and Technology, Government of India for providing research grant. We also acknowledge Department of Biotechnology, Government of India for creating and providing funds for Indian Consortium on Celiac 
disease.

\section{Financial support: None.}

\section{Conflicts of interest: None.}

Author contributions: Srikant Mohta: review of literature and drafting manuscript; Mahendra S Rajput: collecting data and drafting manuscript; Vineet Ahuja: concept design and critical review of the manuscript; and Govind K Makharia: concept design, drafting manuscript, critical review of the manuscript, and overall Guarantee.

\section{References}

1. Lindfors K, Ciacci C, Kurppa K, et al. Coeliac disease. Nat Rev Dis Primer 2019;5:3.

2. Lionetti E, Catassi C. New clues in celiac disease epidemiology, pathogenesis, clinical manifestations, and treatment. Int Rev Immunol 2011;30:219-231.

3. Meeuwisse GW. Diagnostic criteria in coeliac disease. Acta Paediatr Scand 1970;59:461-463.

4. Husby S, Koletzko S, Korponay-Szabó IR, et al. European society for pediatric gastroenterology, hepatology, and nutrition guidelines for the diagnosis of coeliac disease. J Pediatr Gastroenterol Nutr 2012;54:136-160.

5. McMillan SA, Watson RP, McCrum EE, Evans AE. Factors associated with serum antibodies to reticulin, endomysium, and gliadin in an adult population. Gut 1996;39:43-47.

6. Catassi C, Rätsch IM, Fabiani E, et al. Coeliac disease in the year 2000: exploring the iceberg. Lancet 1994;343:200-203.

7. Lionetti E, Gatti S, Pulvirenti A, Catassi C. Celiac disease from a global perspective. Best Pract Res Clin Gastroenterol 2015;29:365-379.

8. Fasano A, Berti I, Gerarduzzi T, et al. Prevalence of celiac disease in atrisk and not-at-risk groups in the United States: a large multicenter study. Arch Intern Med 2003;163:286-292.

9. Hovell CJ, Collett JA, Vautier G, et al. High prevalence of coeliac disease in a population-based study from Western Australia: a case for screening? Med J Aust 2001;175:247-250.

10. Makharia GK, Verma AK, Amarchand R, et al. Prevalence of celiac disease in the northern part of India: a community based study. J Gastroenterol Hepatol 2011;26:894-900.

11. Ramakrishna BS, Makharia GK, Chetri K, et al. Prevalence of adult celiac disease in India: regional variations and associations. Am J Gastroenterol 2016;111:115-123.

12. Shamir R, Lerner A, Shinar E, et al. The use of a single serological marker underestimates the prevalence of celiac disease in Israel: a study of blood donors. Am J Gastroenterol 2002;97:2589-2594.

13. Poddar U, Thapa BR, Nain CK, Prasad A, Singh K. Celiac disease in India: are they true cases of celiac disease? J Pediatr Gastroenterol Nutr 2002;35:508-512.

14. Mohindra S, Yachha SK, Srivastava A, et al. Coeliac disease in Indian children: assessment of clinical, nutritional and pathologic characteristics. J Health Popul Nutr 2001;19:204-208.

15. Bhattacharya M, Kapoor S, Dubey AP. Celiac disease presentation in a tertiary referral centre in India: current scenario. Indian J Gastroenterol 2013;32:98-102.

16. Makharia GK, Baba CS, Khadgawat R, et al. Celiac disease: variations of presentations in adults. Indian J Gastroenterol 2007;26:162-166.

17. Nijhawan S, Katiyar P, Nagaich N, et al. Prevalence of associated disorders in Indian patients with celiac disease. Indian J Gastroenterol 2013;32:330-334

18. Rastogi A, Bhadada SK, Bhansali A, Kochhar R, Santosh R. Celiac disease: a missed cause of metabolic bone disease. Indian J Endocrinol Metab 2012;16:780-785.

19. Sood A, Midha V, Sood N, Avasthi G, Sehgal A. Prevalence of celiac disease among school children in Punjab, North India. J Gastroenterol Hepatol 2006;21:1622-1625.

20. Makharia GK, Verma AK, Amarchand R, et al. Prevalence of celiac disease in the northern part of India: a community based study. J Gastroenterol Hepatol 2011;26:894-900.

21. Keet CA, Matsui EC, Dhillon G, Lenehan P, Paterakis M, Wood RA. The natural history of wheat allergy. Ann Allergy Asthma Immunol 2009; 102:410-415.

22. Igbinedion SO, Ansari J, Vasikaran A, et al. Non-celiac gluten sensitivity: all wheat attack is not celiac. World J Gastroenterol 2017;23:7201-7210.

23. Di Sabatino A, Biagi F, Giuffrida P, Corazza GR. The spectrum of gluten-related disorders. Curr Pediatr Rep 2013;1:182-188.

24. Czaja-Bulsa G, Bulsa M. What do we know now about IgE-mediated wheat allergy in children? Nutrients 2017;9:35.

25. Fasano A, Sapone A, Zevallos V, Schuppan D. Nonceliac gluten sensitivity. Gastroenterology 2015;148:1195-1204.

26. Volta U, Bardella MT, Calabrò A, Troncone R, Corazza GR. An Italian prospective multicenter survey on patients suspected of having non-celiac gluten sensitivity. BMC Med 2014;12:85.

27. Choung RS, Unalp-Arida A, Ruhl CE, Brantner TL, Everhart JE, Murray JA. Less hidden celiac disease but increased gluten avoidance without a diagnosis in the United States: findings from the national health and nutrition examination surveys from 2009 to 2014. Mayo Clin Proc 2017;92:30-38.

28. Singh P, Arora A, Strand TA, et al. Global prevalence of celiac disease: systematic review and meta-analysis. Clin Gastroenterol Hepatol 2018;16:823-836, e2.

29. Yuan J, Zhou C, Gao J, et al. Prevalence of celiac disease autoimmunity among adolescents and young adults in China. Clin Gastroenterol Hepatol 2017;15:572-1579, e1.

30. Lohi S, Mustalahti K, Kaukinen K, et al. Increasing prevalence of coeliac disease over time. Aliment Pharmacol Ther 2007;26:1217-1225.

31. Wu J, Xia B, von Blomberg BM, et al. Coeliac disease: emerging in China? Gut 2010;59:418-419.

32. Wang $\mathrm{XQ}$, Liu $\mathrm{W}, \mathrm{Xu} \mathrm{CD}$, et al. Celiac disease in children with diarrhea in 4 cities in China. J Pediatr Gastroenterol Nutr 2011;53:368-370.

33. Yuan J, Gao J, Li X, et al. The tip of the "celiac iceberg" in China: a systematic review and meta-analysis. PLoS One 2013;8:e81151. 
34. Watanabe C, Komoto S, Hokari R, et al. Prevalence of serum celiac antibody in patients with IBD in Japan. J Gastroenterol 2014;49:825-834.

35. Lu W, Gwee KA, Siah KT, Kang JY, Lee R, Ngan CC. Prevalence of anti-deamidated gliadin peptide antibodies in Asian patients with irritable bowel syndrome. J Neurogastroenterol Motil 2014;20:236241.

36. Ikram MA, Sajid A, Hameed S, Arshad K, Irshad-ul-Haq. Coeliac disease in children presenting with failure to thrive. J Ayub Med Coll Abbottabad 2011;23:6-9.

37. Singh P, Arora S, Singh A, Strand TA, Makharia GK. Prevalence of celiac disease in Asia: a systematic review and meta-analysis. J Gastroenterol Hepatol 2016;31:1095-1101.

38. Zhou C, Gao F, Gao J, et al. Prevalence of coeliac disease in Northwest China: heterogeneity across northern silk road ethnic populations. Aliment Pharmacol Ther 2020;51:1116-1129.

39. Kou GJ, Guo J, Zuo XL, et al. Prevalence of celiac disease in adult Chinese patients with diarrhea-predominant irritable bowel syndrome: a prospective, controlled, cohort study. J Dig Dis 2018;19:136-143.

40. Yap TW, Chan WK, Leow AH, et al. Prevalence of serum celiac antibodies in a multiracial Asian population--a first study in the young Asian adult population of Malaysia. PLoS One 2015;10:e0121908.

41. Fukunaga M, Ishimura N, Fukuyama C, et al. Celiac disease in nonclinical populations of Japan. J Gastroenterol 2018;53:208-214.

42. Zanella S, De Leo L, Nguyen-Ngoc-Quynh L, et al. Cross-sectional study of coeliac autoimmunity in a population of Vietnamese children. BMJ Open 2016;6:e011173.

43. Makharia GK. Celiac disease screening in southern and East Asia. Dig Dis 2015;33:167-174.

44. Makharia GK, Catassi C. Celiac disease in Asia. Gastroenterol Clin North Am 2019;48:101-113.

45. King JA, Jeong J, Underwood FE, et al. Incidence of celiac disease is increasing over time: a systematic review and meta-analysis. Am J Gastroenterol 2020;115:507-525.

46. Okada H, Kuhn C, Feillet H, Bach JF. The 'hygiene hypothesis' for autoimmune and allergic diseases: an update. Clin Exp Immunol 2010;160:1-9.

47. Cataldo F, Montalto G. Celiac disease in the developing countries: a new and challenging public health problem. World J Gastroenterol 2007;13:2153-2159.

48. Kumar P, Joshi PK, Birthal PS. Demand projections for foodgrains in India. Agric Econ Res Rev 2009;22:237-243.

49. Sollid LM, Thorsby E. HLA susceptibility genes in celiac disease: genetic mapping and role in pathogenesis. Gastroenterology 1993;105:910922.

50. Kaukinen K, Partanen J, Mäki M, Collin P. HLA-DQ typing in the diagnosis of celiac disease. Am J Gastroenterol 2002;97:695-699.

51. Lundin KE, Sollid LM, Qvigstad E, et al. T lymphocyte recognition of a celiac disease-associated cis- or trans-encoded HLA-DQ alpha/betaheterodimer. J Immunol 1990;145:136-139.

52. Tollefsen S, Arentz-Hansen H, Fleckenstein B, et al. HLA-DQ2 and -DQ8 signatures of gluten T cell epitopes in celiac disease. J Clin Invest 2006;116:22262236.

53. Tjon JM, van Bergen J, Koning F. Celiac disease: how complicated can it get? Immunogenetics 2010;62:641-651.

54. Stepniak D, Koning F. Celiac disease--sandwiched between innate and adaptive immunity. Hum Immunol 2006;67:460-468.

55. Lindfors K, Koskinen O, Kaukinen K. An update on the diagnostics of celiac disease. Int Rev Immunol 2011;30:185-196.

56. Cummins AG, Roberts-Thomson IC. Prevalence of celiac disease in the Asia-Pacific region. J Gastroenterol Hepatol 2009;24:1347-1351.

57. Ludvigsson JF, Leffler DA, Bai JC, et al. The Oslo definitions for coeliac disease and related terms. Gut 2013;62:43-52.

58. Catassi C, Elli L, Bonaz B, et al. Diagnosis of non-celiac gluten sensitivity (NCGS): the salerno experts' criteria. Nutrients 2015;7:4966-4977.

59. Catassi C, Alaedini A, Bojarski C, et al. The overlapping area of nonceliac gluten sensitivity (NCGS) and wheat-sensitive irritable bowel syndrome (IBS): an update. Nutrients 2017;9:1268.

60. Irvine AJ, Chey WD, Ford AC. Screening for celiac disease in irritable bowel syndrome: an updated systematic review and meta-analysis. Am J Gastroenterol 2017;112:65-76.

61. Makharia A, Catassi C, Makharia GK. The overlap between irritable bowel syndrome and non-celiac gluten sensitivity: a clinical dilemma. Nutrients 2015;7:10417-10426.

62. Nanda R, James R, Smith H, Dudley CR, Jewell DP. Food intolerance and the irritable bowel syndrome. Gut 1989;30:1099-1104.

63. Vazquez-Roque MI, Camilleri M, Smyrk T, et al. A controlled trial of gluten-free diet in patients with irritable bowel syndrome-diarrhea: effects on bowel frequency and intestinal function. Gastroenterology 2013;144:903-911, e3.

64. Skodje G, Sarna VK, Minelle IH, et al. Fructan, rather than gluten, induces symptoms in patients with self-reported non-celiac gluten sensitivity. Gastroenterology 2018;154:529-539, e2.

65. Dionne J, Ford AC, Yuan Y, et al. A systematic review and meta-analysis evaluating the efficacy of a gluten-free diet and a low FODMAPs diet in treating symptoms of irritable bowel syndrome. Am J Gastroenterol 2018;113:1290-1300.

66. Gibson PR, Muir JG. Not all effects of a gluten-free diet are due to removal of gluten. Gastroenterology 2013;145:693.

67. Biesiekierski JR, Peters SL, Newnham ED, Rosella O, Muir JG, Gibson PR. No effects of gluten in patients with self-reported nonceliac gluten sensitivity after dietary reduction of fermentable, poorly absorbed, short-chain carbohydrates. Gastroenterology 2013;145:320328, e1-e3.

68. Volta U, Pinto-Sanchez MI, Boschetti E, Caio G, De Giorgio R, Verdu EF. Dietary triggers in irritable bowel syndrome: is there a role for gluten? J Neurogastroenterol Motil 2016;22:547-557.

69. Pinto-Sanchez MI, Bercik P, Verdu EF. Motility alterations in celiac disease and non-celiac gluten sensitivity. Dig Dis 2015;33:200-207.

70. Makharia GK, Mulder CJ, Goh KL, et al. Issues associated with the emergence of coeliac disease in the Asia-Pacific region: a working party report of the world gastroenterology organization and the Asian Pacific Association of Gastroenterology. J Gastroenterol Hepatol 2014;29:666677.

71. Singh P, Singh A, Silvester JA, et al. Inter- and intra-assay variation in the diagnostic performance of assays for anti-tissue transglutaminase in 2 
populations. Clin Gastroenterol Hepatol Online First: 20 Sep 2019. doi: 10.1016/j.cgh.2019.09.018

72. See JA, Kaukinen K, Makharia GK, Gibson PR, Murray JA. Practical insights into gluten-free diets. Nat Rev Gastroenterol Hepatol
2015;12:580-591.

73. Mehtab W, Singh N, Malhotra A, Makharia GK. All that a physician should know about gluten-free diet. Indian J Gastroenterol 2018;37:392401. 